\title{
Reversible Loss of Hippocampal Function in a Mouse Model of Demyelination/Remyelination
}

\author{
Aniruddha Das', Chinthasagar Bastian', Lexie Trestan', Jason Suh', Tanujit Dey², \\ Bruce D. Trapp ${ }^{1,3}$, Selva Baltan ${ }^{1 \dagger}$ and Hod Dana ${ }^{1,3 *}$ \\ ${ }^{1}$ Department of Neurosciences, Lerner Research Institute, Cleveland Clinic, Cleveland, $\mathrm{OH}$, United States, ${ }^{2}$ Department \\ of Quantitative Health Sciences, Lerner Research Institute, Cleveland Clinic, Cleveland, $\mathrm{OH}$, United States, ${ }^{3}$ Department \\ of Molecular Medicine, Cleveland Clinic Lerner College of Medicine, School of Medicine, Case Western Reserve University, \\ Cleveland, $\mathrm{OH}$, United States
}

OPEN ACCESS

Edited by:

Shai Berlin,

Technion-Israel Institute

of Technology, Israel

Reviewed by:

Lorenzo Di Cesare Mannelli,

University of Florence, Italy

Maarten H. P. Kole,

Netherlands Institute for Neuroscience

(KNAW), Netherlands

Chao Zhao,

University of Cambridge,

United Kingdom

*Correspondence:

Hod Dana

danah@ccf.org

tPresent address:

Selva Baltan,

Department of Anesthesiology and Perioperative Medicine, Oregon Health \& Science University, Portland,

OR, United States

Specialty section:

This article was submitted to

Cellular Neuropathology,

a section of the journal

Frontiers in Cellular Neuroscience

Received: 26 September 2019

Accepted: 24 December 2019

Published: 22 January 2020

Citation:

Das A, Bastian C, Trestan L, Suh J, Dey T, Trapp BD, Baltan S and

Dana H (2020) Reversible Loss of Hippocampal Function in a Mouse

Model

of Demyelination/Remyelination.

Front. Cell. Neurosci. 13:588.

doi: 10.3389/fncel.2019.00588
Demyelination of axons in the central nervous system (CNS) is a hallmark of multiple sclerosis (MS) and other demyelinating diseases. Cycles of demyelination, followed by remyelination, appear in the majority of MS patients and are associated with the onset and quiescence of disease-related symptoms, respectively. Previous studies in human patients and animal models have shown that vast demyelination is accompanied by wide-scale changes to brain activity, but details of this process are poorly understood. We used electrophysiological recordings and non-linear fluorescence imaging from genetically encoded calcium indicators to monitor the activity of hippocampal neurons during demyelination and remyelination over a period of 100 days. We found that synaptic transmission in CA1 neurons was diminished in vitro, and that neuronal firing rates in CA1 and the dentate gyrus (DG) were substantially reduced during demyelination in vivo, which partially recovered after a short remyelination period. This new approach allows monitoring how changes in synaptic transmission induced by cuprizone diet affect neuronal activity, and it can potentially be used to study the effects of therapeutic interventions in protecting the functionality of CNS neurons.

Keywords: multiple sclerosis, two-photon microscopy, calcium imaging, electrophysiology, cuprizone

\section{INTRODUCTION}

Multiple sclerosis (MS) is an inflammatory demyelinating disease of the central nervous system (CNS) that affects more than 2.3 million patients worldwide (Wallin et al., 2019). For approximately $85 \%$ of patients, disease progression begins with a "relapsing-remitting" phase, where symptoms including motor deficits and vision loss that correlate with increased formation of demyelinated lesions detected by magnetic resonance imaging (MRI). Over time, these symptoms resolve into periods of clinical quiescence and partial remyelination of demyelinated axons (Chari, 2007; Goldenberg, 2012). In the majority of MS patients, the disease course eventually transforms from the relapsing-remitting phase to a secondary-progressive phase, where symptoms continually worsen (Mahad et al., 2015). Traditionally, MS was considered to be mainly a white-matter disease, but today it is widely accepted that there is also a critical gray-matter component, including demyelination of the cerebral cortex and deep gray matter (Geurts and Barkhof, 2008). Previous studies have also identified changes in brain activity patterns of MS patients during both the 
relapsing-remitting and secondary-progressive forms of the disease, where presumably the reduced activity of MS-affected brain regions is partially compensated for by increased activity in other brain regions (Staffen et al., 2002; Chiaravalloti and DeLuca, 2008).

Studying the relationships between demyelination/ remyelination cycles, motor and cognitive deficits, and changes in brain activity patterns requires the use of appropriate animal models and proper experimental methods. A commonly used mouse model for studying demyelination and remyelination is the cuprizone model (Kipp et al., 2009; Vega-Riquer et al., 2019), in which the copper chelator cuprizone is added to the mouse diet, resulting in damage to oligodendrocyte cells in the CNS. Over time, cuprizone causes extensive demyelination in various brain areas, including the hippocampus, corpus callosum, cortex, and cerebral white matter, which is partially reversible upon cessation of cuprizone by returning the animals to normal diet (Skripuletz et al., 2008; Kipp et al., 2009; Koutsoudaki et al., 2009; Sachs et al., 2014). Interestingly, cuprizone-treated mice, similar to MS patients, were identified as having demyelinationinduced reorganization of brain circuitry (Huebner et al., 2017). Moreover, the extensive brain demyelination produced by cuprizone resulted in significantly poorer performance in various behavioral tests, including hippocampus-dependent tasks (Xu et al., 2009). This reduction in performance was reversed upon remyelination (Dutta et al., 2013). Therefore, the cuprizone model seems to capture the demyelination-induced brain-wide activity reorganization that has been identified in MS patients. However, the traditional brain mapping methods used to identify these brain-wide activity changes in living mice were usually functional MRI and electroencephalogram (EEG), which have limited spatial and temporal resolutions that restrict their ability to assess rapid and local changes in brain activity. In order to uncover demyelination-induced effects on single cells and local networks, a method is required that allows micron-scale identification and single action potential (AP) detection in vivo.

Substantial improvements in spatiotemporal resolution can be achieved by using non-linear optics to detect brain activity (Svoboda and Yasuda, 2006). Two-photon laser scanning microscopy (TPLSM) of the fluorescence signal from genetically encoded calcium indicators (GECIs) enables monitoring the activity of thousands of individual neurons in the rodent brain with micron-scale resolution and high signal-to-noise ratio (Chen et al., 2013; Dana et al., 2016, 2019). Moreover, state-ofthe-art GECIs provide the temporal dynamics and sensitivity that are necessary to allow detection of single APs over time scales of months, mainly by switching to stable transgenic expression strategies (Dana et al., 2014, 2016, 2018, 2019; Madisen et al., 2015; Wekselblatt et al., 2016). Therefore, this approach may enable the exploration of changes in brain activity following demyelination and remyelination with the required sensitivity and accuracy to detect changes in individual neurons over time.

In this study, we combined electrophysiology and TPLSM recording of acute and chronic activity patterns of neurons in the mouse hippocampus before, during, and after mice were fed with cuprizone diet. We identified rapid degeneration of CA1 activity following the initiation of cuprizone diet, which were faster than expected by the induced demyelination alone, which decreased neuronal activity to $\sim 20 \%$ of its pre-cuprizone level. We also found that synaptic transmission in CA1 neurons in response to sustained pre-synaptic stimuli was drastically diminished after the initiation of cuprizone diet. Synaptic transmission and neuronal activity partially recovered upon cessation of cuprizone diet. Thus, our study presents a new experimental approach for studying the effects of toxin-induced changes to brain physiology, such as demyelination and remyelination, on the integrity of individual neurons and neuronal circuits, and it also provides a platform to test the benefits of potential treatment options.

\section{MATERIALS AND METHODS}

All surgical and experimental procedures were conducted in accordance with protocols approved by the Lerner Research Institute, Institutional Animal Care and Use Committee and Institutional Biosafety Committee.

\section{Hippocampal Slice Electrophysiology}

Electrophysiological experiments were performed in the CA1 region of $400 \mu \mathrm{m}$-thick transverse hippocampal slices as reported previously (Tekkok and Krnjevic, 1995; Provencio et al., 2016). Briefly, a cohort of 46 mice was divided into control $(n=18$, normal diet, daily Rapamycin injections) and experimental groups ( $n=28$, cuprizone diet with daily Rapamycin injections). The experimental group was further divided and, synaptic transmission was monitored and quantified beginning at 1 $(n=2), 3(n=9), 4(n=8), 5(n=3)$, and 6 weeks $(n=3)$ of cuprizone diet. A final group consisted of mice on cuprizone diet for 6 weeks, which were then returned to control diet for 6 weeks to remyelinate $(n=3,6 \mathrm{~W} \mathrm{DM}+6 \mathrm{~W}$ RM). Synaptic transmission was recorded in a subset of Controls at week 0 $(n=8)$ and beginning at $4(n=5,4 \mathrm{~W}$ C) or 6 weeks $(n=5$, $6 \mathrm{~W} \mathrm{C)}$ to match the respective experimental groups. Mice were decapitated after anesthesia and brains were immediately removed and placed in ice-cold $\left( \pm 4^{\circ} \mathrm{C}\right)$ artificial cerebrospinal fluid (ACSF) containing in mM: $126 \mathrm{NaCl}, 3.5 \mathrm{KCl}, 1.3 \mathrm{MgCl}_{2}$, $2 \mathrm{CaCl}_{2}, 1.3 \mathrm{NaH}_{2} \mathrm{PO}_{4}, 25 \mathrm{NaHCO}_{3}$, and 10 glucose at $\mathrm{pH}$ 7.4. Hippocampi were quickly dissected and sliced with a McIlwain tissue chopper. Transverse slices were then transferred to ACSF solution and were continuously bubbled with 95\% $\mathrm{O}_{2} / 5 \% \mathrm{CO}_{2}$ for at least $1-2 \mathrm{~h}$ at room temperature to stabilize.

Individual slices were then transferred and placed on a Haastype tissue chamber (Warner Instruments, Harvard Apparatus) on a custom mesh (Warner Instruments, Catalog \# 99-1129) to avoid air bubble accumulation and were stabilized using a slice holder (Warner Instruments, Cat. \# 64-1415). Slices were kept at the interface of a moist humidified gas mixture of 95\% $\mathrm{O}_{2} / 5 \% \mathrm{CO}_{2}$ and warm ACSF (at $33-34^{\circ} \mathrm{C}$ ) bubbled with 95\% $\mathrm{O}_{2} / 5 \% \mathrm{CO}_{2}$ continually at a flow rate of $3-3.5 \mathrm{ml} / \mathrm{min}$. For simultaneous extracellular recordings of afferent volleys (AV) and excitatory post-synaptic potentials (EPSPs), glass microelectrodes with 1-micron tips were pulled (Sutter Instruments, Borosilicate Glass with filament) and filled with $2 \mathrm{M} \mathrm{NaCl}$ (resistance 
1-2 M $\Omega$ ). Electrode tips were polished using a Micro-Forge (Leica, MF-830, Narishige, Japan) and placed in the CA1 region of stratum radiatum layer to record synaptic transmission. Responses were evoked by stimulation of Schaffer collateral commissural fibers by a custom-designed bipolar tungsten wire electrode (Microprobes Part\# PI2ST30.1B3) with $50 \mu \mathrm{s}-$ long pulses at 30-s intervals. After stabilization, synaptic transmission was recorded for at least $30 \mathrm{~min}$ as baseline. Changes in synaptic transmission properties were quantified by measuring the peak amplitude of EPSPs, as well as their area (Clampfit 10.7) to account for smaller slower responses. Maximum EPSPs, evoked by $100 \%$ of the stimulation amplitude $(1 \mathrm{~mA})$, were chosen for these measurements.

\section{Installation of Hippocampal Windows}

For hippocampal window implantation, we slightly modified a previously described procedure (Dombeck et al., 2010; Lee et al., 2019). Thy1-jRGECO1a line 8.31 mice were anesthetized using isoflurane $(2.5-3 \%$ for induction, $1.5 \%$ during surgery) on a $37^{\circ} \mathrm{C}$ heating pad. Local pain medication (bupivacaine $0.5 \%$ ) was injected into the craniotomy area before the skin and connective tissue were removed to expose the skull. A circular craniotomy (3.2 $\mathrm{mm}$ diameter) was drilled using a hand drill (OMNIDRILL35, World Precision Instruments). The cortex and corpus callosum, but not the alveus above the dorsal part of the hippocampus, were removed by gentle suction with saline using 26G and 28G sharp and blunt needles connected to a vacuum line. After the hippocampus was exposed, it was kept in place by gently pressing a glass coverslip to it $(3 \mathrm{~mm}$ diameter \#1 glass, Warner Instruments) attached to a metal cannula (2.65 $\mathrm{mm}$ internal diameter, $3.2 \mathrm{~mm}$ outer diameter, $1.8 \mathrm{~mm}$ length, New England Small Tube Corp., type 304L stainless steel). The cannula was attached to the skull bone using dental cement (Contemporary Ortho-Jet, Lang Dental). A custom stainlesssteel head post was cemented to the skull using the same dental cement.

\section{Recording of Hippocampal Activity}

Seven to fourteen days after the craniotomy surgery, we started monitoring hippocampal activity. The same procedure was followed for all mice before, during, and after the cuprizone diet period. Mice were anesthetized (3\% isoflurane) and injected with Chlorprothixene Hydrochloride (IM, $30 \mu \mathrm{l}$ of a $0.33 \mathrm{mg} / \mathrm{ml}$ solution, Santa Cruz). Recordings started at least 30 min after injection. Mice were put on a $37^{\circ} \mathrm{C}$ heating pad in the dark, and isoflurane levels were reduced to $0.5-0.75 \%$ prior to recording to bring the mice to a lightly anesthetized state, where they do not move but are sensitive to pain. Spontaneous activity was recorded using 20-120 mW of $1100 \mathrm{~nm}$ excitation light (Insight X3, Spectra-Physics) and a resonant-scanner two-photon microscope ( $512 \times 512$ pixels, $30 \mathrm{~Hz}$ acquisition rate, Bergamo II, Thorlabs). Light intensity was controlled using a Pockels Cell (model 350105 , Conoptics) to maintain similar signal-to-noise ratios across different animals. Data were recorded on a weekly basis from all animals, and each recording session included acquisition of $200 \mathrm{~s}$ for each field of view (FOV), 3-5 FOVs from CA1 and 2-4 FOVs from dentate gyrus (DG) for each mouse.

\section{Data Analysis}

Data analysis was performed using custom MATLAB scripts (MathWorks). Every two frames in the recorded time series were averaged to improve the signal-to-noise ratio, reducing the acquisition rate to $15 \mathrm{~Hz}$. Small drifts and movements of the imaged area during the $200 \mathrm{~s}$ recording time were corrected with a MATLAB script using the TurboReg plug-in of ImageJ (Thevenaz et al., 1998). Ring-shaped regions of interest (ROI) corresponding to all identifiable somata were selected using a semi-automatic graphical user interface (Chen et al., 2013). Fluorescence signal from all pixels inside of a ROI were averaged to calculate the fluorescence signal $(\mathrm{F})$ for every cell. Baseline fluorescence levels $\left(\mathrm{F}_{0}\right)$ for each ROI were estimated as its $\mathrm{F}$ median value across the $200 \mathrm{~s}$ of recording. To estimate AP firing from the fluorescence data, we calculated $\Delta \mathrm{F} / \mathrm{F}_{0}=\left(\mathrm{F}-\mathrm{F}_{0}\right) / \mathrm{F}_{0}$ for each cell, and fed it to a published model (Deneux et al., 2016) for extracting AP activity from calcium traces (for accepting the model assumption, a trace of $1+\Delta \mathrm{F} / \mathrm{F}_{0}$ was used). We fit the model with the following parameters, according to jRGECO1a published characterization (Dana et al., 2016): one AP amplitude of $15 \%$, decay time of $175 \mathrm{~ms}$, and Hill coefficient of 1.9. Saturation level was set to 0.001 and signal drift to 0.03 . Noise level was estimated using the model's internal function. The model results were inspected visually for accuracy, and for CA1 data the noise level estimation was increased by 50\% to lower the false-positive rate. We identified active cells as cells with at least one identified AP by the AP-extraction model. The average activity was calculated as the total number of APs divided by the recording duration of $200 \mathrm{~s}$. An activity burst was defined as firing of at least five APs within $660 \mathrm{~ms}$.

For calculating the cellular contrast, we averaged all pixels in the ring-shaped ROI (covering the cellular cytosol), and averaged $5 \times 5$ grid of pixels around the ROI center, corresponding to an area inside the nucleus. The contrast was calculated as the difference between the averaged cytosolic and nucleic signals, divided by the nucleic signal.

\section{Statistical Analysis}

Statistical analysis was performed using MATLAB and R software (version 3.5.1) ${ }^{1}$. We performed four different statistical tests to identify changes in activity across different recording dates and mice. Wilcoxon Rank Sum tests were used to compare changes in median firing rate between two different recording dates from the same mice. Mann-Kendall Trend Tests were used to identify any monotonic trends in median firing rates during the cuprizone diet period (eight recording dates) for individual mice. Paired $t$-tests were used to compare changes in median firing rates of cuprizone or control groups across two recording dates. A repeated measures linear mixed effects model (repeated ANOVA test) was performed to determine the interaction of group (cuprizone and control) and time (eight recording dates) on CA1 and DG median activity rates, separately. The level of significance was set to $P<0.05$ for all hypothesis testing procedures.

\footnotetext{
${ }^{1}$ https://cran.r-project.org
} 
For analysis of in vitro hippocampal experiments, all means are reported \pm SEM and significant differences within a group was assessed by a one-way ANOVA, followed by Bonferroni's test. $\mathrm{N}$ denotes animal numbers in the text and parentheses in Figure 1 indicate slice numbers.

\section{Mouse Model of Demyelination and Remyelination}

After the mice recovered from craniotomy surgery and we recorded at least one imaging session from them (7-14 days after the craniotomy surgery), we switched their diet to include $0.3 \%$ cuprizone (TD.140805, Envigo). Mice were provided access to food ad libitum. Food pellets were refrigerated and were replaced three times per week to ensure cuprizone efficiency. We continued to monitor hippocampal activity on a weekly basis. After 53 days of cuprizone diet, we identified that activity levels had reached a median level of zero APs (Figure 2B) in one of the mice, and we switched the mouse diet back to normal food. Mouse weight was monitored before each recording session (Supplementary Table 1). For electrophysiological recording experiments, mice were also injected daily with rapamycin for 12 weeks.

\section{RESULTS}

\section{Demyelination Interrupts Synaptic Transmission in Hippocampal CA1 Neurons}

Previous studies have identified that the hippocampus is severely demyelinated following 6-12 weeks of cuprizone diet, and that additional injections of rapamycin have eliminated the spontaneous remyelination that occurs when mice are fed with cuprizone (Sachs et al., 2014; Bai et al., 2016). These mice performed poorly in a hippocampus-dependent spatial-memory task (Dutta et al., 2013). Therefore, to investigate changes in neuronal activity in the hippocampus, we conducted a set of in vitro experiments in acute hippocampal slices. Fortysix mice were divided into seven cohorts and controls. The groups included $1,3,4,5$, or 6 weeks of cuprizone-dietinduced demyelination and daily rapamycin injections, and one group with 6 weeks of cuprizone diet with rapamycin injections, followed by an additional 6 weeks of remyelination with regular diet and no rapamycin injections (Figure 1). The control group received regular diet and daily rapamycin injections for 6 weeks, and recordings were performed at 0,4 , or 6 weeks to compare synaptic properties between demyelination and rapamycin-only conditions (Supplementary Figure 1). EPSPs together with clear AVs were evoked to monitor axon function in Schaeffer collateral commissural fibers. A successful synaptic transmission is a result of presynaptic input and a subsequent synaptic response. Sample traces shown in Figure 1 denote that in every control slice following the stimulus artifact (blue line, due to square pulse delivered with the stimulation electrode), a prominent AV and EPSP were evoked (Figure 1A, $0 \mathrm{~W}$ C, red *: AVs, black arrow for EPSPs). EPSPs presented

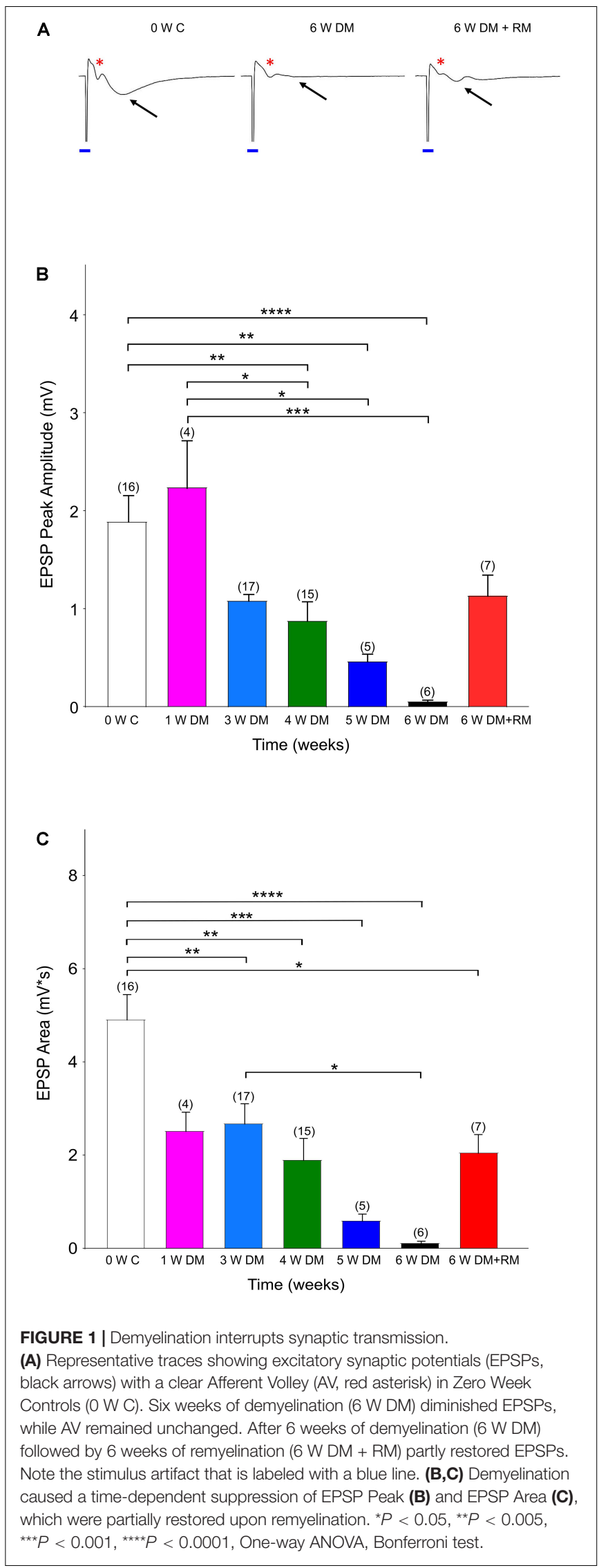


A

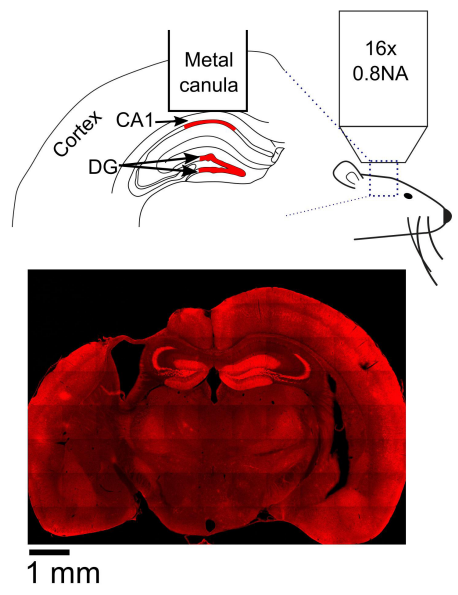

B

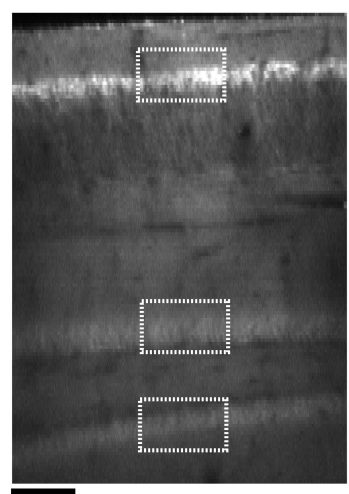

$\overline{100 \mu \mathrm{m}}$
C

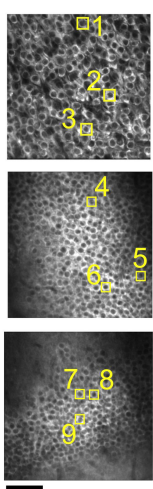

$\overline{50} \mu \mathrm{m}$
D

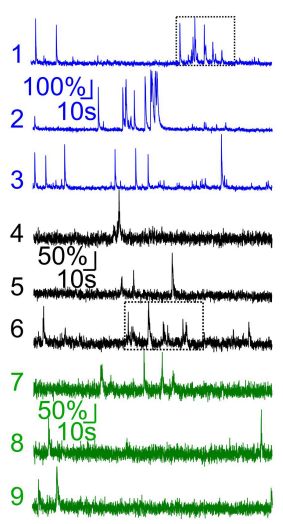

E

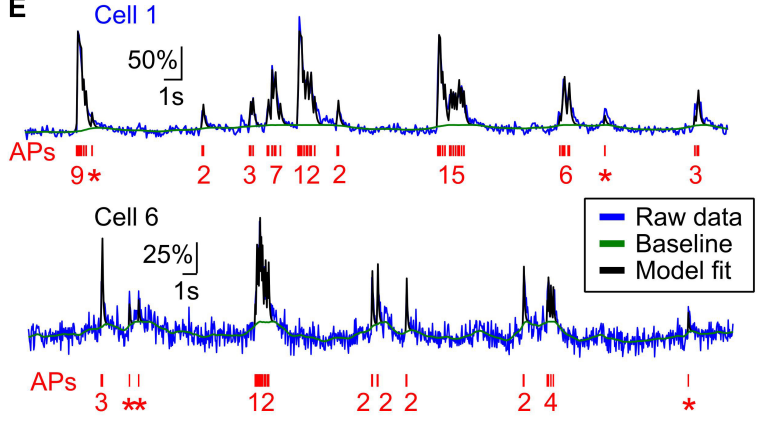

$\mathbf{F}$

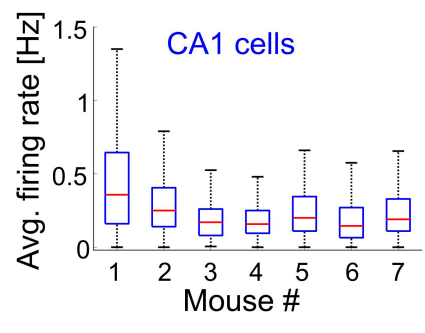

G

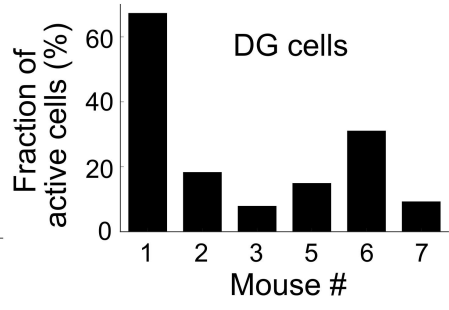

FIGURE 2 | Optical recording from mouse CA1 and DG regions of the hippocampus. (A) Top, schematic illustration of the mouse hippocampal recording experiments. We implanted metal cannulas with a glass bottom on top of the hippocampi of transgenic Thy-jRGECO1a mice to gain optical access to CA1 and DG neurons (shown in red). Hippocampus geometry was adopted from a mouse brain atlas (Dong, 2008). Bottom, a coronal section from the brain of mouse 1 shows the location of the hippocampal window and native jRGECO1a fluorescence signal throughout the brain. Note that some distortion to the brain tissue occurred when the metal cannula was detached from it. (B) In vivo dorsal-to-ventral view of a Thy1-jRGECO1a line 8.31 transgenic mouse expressing the red GECl jRGECO1a in the hippocampus 3 weeks after a craniotomy surgery. The tissue on top of the hippocampal formation was removed to expose the hippocampus and a $z$-stack series of two-photon microscopy images shows CA1 and DG regions. White rectangles indicate the location of the CA1 and DG (upper and lower blades) projection neurons, labeled by jRGECO1a. (C) Images of CA1 pyramidal cells (top image) and DG granular cells from the upper and lower blades of DG (middle and bottom images, respectively), corresponding to the respective rectangles shown in panel (B). (D) $\Delta F / F_{0}$ traces of the nine cells highlighted with yellow squares in panel (C). Note the differences in firing pattern and amplitude between the CA1 and DG cells. (E) Model fit (Deneux et al., 2016) (black line) to the raw fluorescence signal (blue line) of cells one and six [upper and lower traces, respectively, corresponding to the rectangles shown in panel (D)] to extract the baseline signal (green line) and AP firing (red, bottom, single AP events are represented with asterisks). The model was fed with jRGECO1a biophysical parameters (Dana et al., 2016), and showed good agreement to the recorded data from both CA1 (top) and DG (bottom) neurons. (F) Summary of average firing rates of all recorded CA1 pyramidal cells from seven mice before the initiation of cuprizone diet (328-623 cells from a single recording session for each mouse, 3,167 cells total). Red lines correspond to medians, blue boxes show the 25th-75th percentile range, whisker length is the shorter of 1.5 times the 25th-75th range or the extreme data point. Outliers are not shown. (G) Fraction of active DG neurons from all recorded DG neurons from six mice before the start of cuprizone diet (470-997 cells from a single recording session for each mouse, DG cells of mouse 4 were not visible, 3,113 cells total).

with a peak amplitude of $\sim 2 \mathrm{mV}$ and an average area of $5 \mathrm{mV} \times \mathrm{s}$ (Figure 1B, white columns, $n=16)$. As demyelination continued, EPSP peak amplitude and area gradually started to decline, becoming prominently low by 3-4 weeks (Figures 1A,B, blue and green columns, $n=17$ and 15 , respectively) and almost completely diminishing by 6 weeks (Figures 1A,B, black columns, $n=6$ ). These changes were specific to cuprizone treatment because recordings in 4- and 6-week control groups were similar to 0-week control values (Supplementary Figure 1). Remyelination partially restored EPSPs, albeit with smaller amplitude compared to the control groups (Figures 1A-C, $6 \mathrm{~W} \mathrm{DM}+\mathrm{RM}, n=7)$. Therefore, demyelination effectively interrupted synaptic transmission, characterized by loss of EPSPs, despite sustained presynaptic input, while remyelination partially restored synaptic transmission.

\section{Chronic Optical Recording of Neuronal Activity From CA1 and DG Cells of Thy1-jRGEC01a Mice}

In order to further investigate the prevalence and time course of CA1 neuronal activity modulations, we conducted additional experiments for longitudinal optical recording of GECI signal from the hippocampus of lightly anesthetized mice $(n=7$ mice, 
10-17 weeks old when implanted with an optical window; mice 1, 4, and 7 were males; mice 2, 3, 5, and 6 were females). Optical recording from CA1 cells usually requires removal of the cortical tissue on top of the recorded area (Dombeck et al., 2010; Ziv et al., 2013; Lee et al., 2019). Recordings from the DG area have also required removal of the CA1 region, causing severe damage to the hippocampal circuit (Danielson et al., 2016, 2017). Recent developments in the field of three-photon functional microscopy offer deeper tissue penetration to depths greater than $1 \mathrm{~mm}$, enabling the recording of CA1 cells under an intact cortex (Ouzounov et al., 2017); however, this method is not yet able to probe deeper structures such as the DG. To allow deep-hippocampal recording while minimizing disruption to its integrity, we followed previous studies that used red GECIs to facilitate deeper TPLSM imaging, which allows recording from the DG without removing the CA1 layer (Dana et al., 2016; Pilz et al., 2016). We used a recently developed transgenic line, Thy1-jRGECO1a line GP8.31, which expresses the state-of-theart red GECI jRGECO1a under the Thyl promoter. jRGECO1a is expressed mostly in projection neurons across various brain regions, including the CA1 pyramidal and DG granular layers of the hippocampus (Dana et al., 2018). The enhanced imaging depth and high sensitivity achieved with jRGECO1a compared to green GECIs like GCaMP that we previously used for cortical imaging (Dana et al., 2016) enabled us to record, in six out of the seven mice used in this study, from the upper and lower blades of the DG down to $650 \mu \mathrm{m}$ under the hippocampal surface using up to $120 \mathrm{~mW}$ of $1100 \mathrm{~nm}$ excitation light (Figures 2A-D).

Recording of spontaneous activity from CA1 and DG neurons of lightly anesthetized mice revealed different firing patterns across the two populations (Figure 2D and Supplementary Movies 1, 2). To quantify activity patterns from the recorded fluorescence traces, we used a physiological model for extracting AP firing from calcium signals (Figure 2E) (Deneux et al., 2016). The majority of CA1 cells fired bursts of APs with a median average firing rate of $0.21 \mathrm{~Hz}$ and a maximal instantaneous firing rate of $8.15 \mathrm{~Hz}(3,114 / 3,167$ CA1 cells were detected with at least firing of one AP; 2,099/3,167 cells fired bursts of APs, instantaneous firing rate was measured for 1-s time bins. Data from $n=7$ mice, one recording session for each mouse, Figure 2F). Most DG cells were not active during our recordings, and firing rates were substantially lower than those of CA1 cells (1,048/3,113 DG cells were detected with at least firing of one AP; only 184 cells from which were detected with average firing rate higher than $0.05 \mathrm{~Hz} ; n=6$ mice, one recording session for each mouse, Figure 2G).

Chronic activity recording from DG neurons proved to be more challenging than CA1 recording, since the deeper location of the DG resulted in a lower signal-to-noise ratio, caused by attenuation of the incoming laser beam and outgoing fluorescence signal. Moreover, we found that in some of the mice, the recorded image quality gradually degraded 6-12 weeks after cranial window implantation (Supplementary Figure 2, the DG of mouse 4 could not be recorded, the contrast of mouse 3's DG neurons deteriorated 1 month after the craniotomy surgery, and was excluded from the rest of the study), resulting in reduced sensitivity to AP detection, or even an inability to identify DG cells. We quantified this degeneration by measuring the contrast between the cytosolic jRGECO1a signal and the nuclear signal, which should be much weaker in jRGECO1a-expressing neurons (Dana et al., 2016). The contrast of CA1 neurons was 2-5 times higher than DG neurons in the same animal. Data from DG neurons, recorded on dates with very low contrast, were excluded from our analysis (Supplementary Figure 2B).

\section{Cuprizone Diet Reduced CA1 Projection Neuron Firing Rates}

To explore the reversible change in CA1 excitability (Figure 1) and to quantify how cuprizone-induced effects, such as demyelination and remyelination, affect the activity of single neurons in the hippocampus, we fed $n=4$ mice with $0.3 \%$ cuprizone diet ( $n=3$ mice for 53 days, mouse 4 optical window quality deteriorated after 16 days of cuprizone diet so it was subsequently excluded from the rest of the study, Figure 3A). A control group of $n=3$ mice was implanted with a hippocampal window and received normal diet. These mice were not injected with Rapamycin in order to allow for faster remyelination after the end of the cuprizone diet period (Sachs et al., 2014). Weekly monitoring of CA1 and DG activity was conducted before, during, and after the cuprizone diet period, in order to identify changes to activity patterns in the same animal. Shortly after the initiation of the cuprizone diet, the average firing rate of CA1 cells was significantly decreased by $57 \%$ (comparing the mean of median firing rate values per mouse of $n=4$ mice, with 1,695 and 1,823 identified neurons on days 0 and 9 for cuprizone diet, respectively, $P<10^{-13}$ for the decrease in each mouse firing rate, Wilcoxon Rank Sum Test; $P=0.02$, paired $t$-test between median firing rates of mice $1-4$ on days 0 and 9; Figures 3B,C). During the 53 days of cuprizone diet, CA1 median firing rate significantly decreased for individual mice ( $P=0.002,0.064,0.035$, Mann-Kendall Trend Test for mice 1 , 2, and 3, respectively, see examples in Supplementary Figure 3), but the group effect was not significant, presumably because of the small sample size $(P=0.07$, paired $t$-test for change for median firing rates of mice 1-3). We also detected shortterm increases in firing rates (Figures 3B,C, days 20-30) that might be associated with partial spontaneous remyelination, as was previously identified in cuprizone-treated mice (Sachs et al., 2014). On the last day of cuprizone diet, the average firing rate was significantly reduced to $16.5 \%$ of pre-diet levels (comparing the mean of median firing rate values per mouse of $n=3$ mice, with 1,135 and 1,356 identified neurons on days 0 and 53 for cuprizone diet, respectively. $P<10^{-36}$, Wilcoxon Rank Sum Test, Supplementary Movies 1, 3). A similar decrease was identified in the fraction of cells that fired bursts on APs (Figure 3D, Methods). Monitoring CA1 activity of $n=3$ control mice showed no similar decrease. Changes in firing rates and burst activity were smaller than in the cuprizone group, had no identified monotonous trend, and presumably were the result of differences in anesthesia levels and brain state (Figures 3E-G and Supplementary Movies 4, 5; $P=0.99$, 0.86, 0.14, MannKendall Trend Test for mice 5, 6, and 7, respectively; $P=0.53$ and 0.49 , paired $t$-test for changes of median firing rates of mice 
A
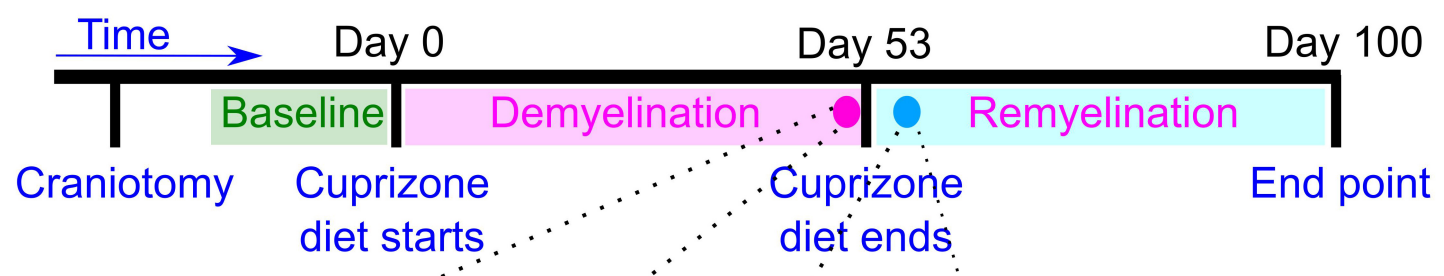

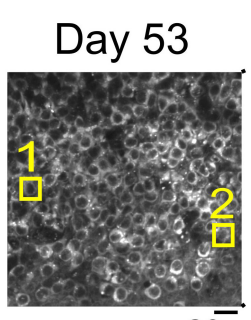

$2 \bar{\mu} \mathrm{m}$

B

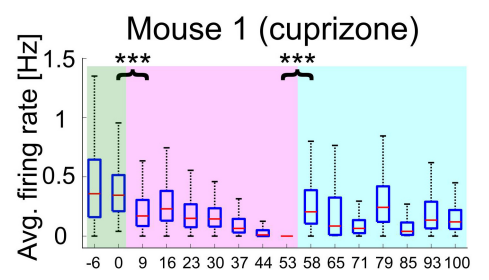

Day

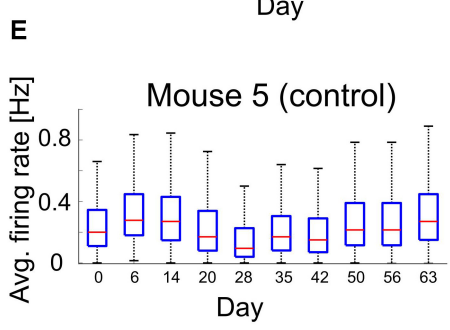

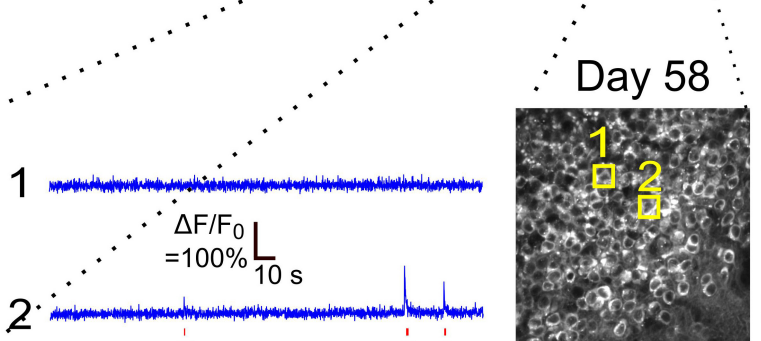

8 ' 4

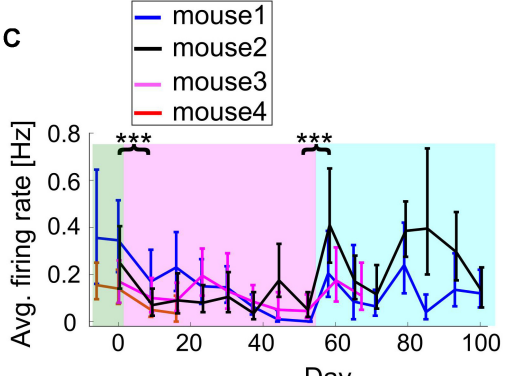

Day

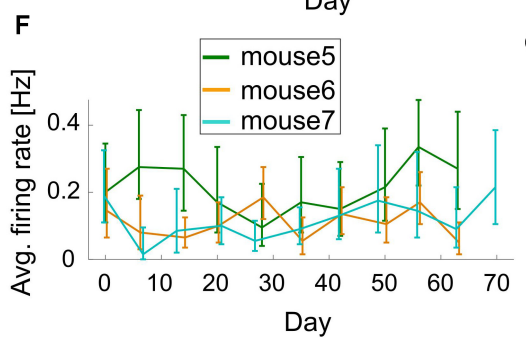

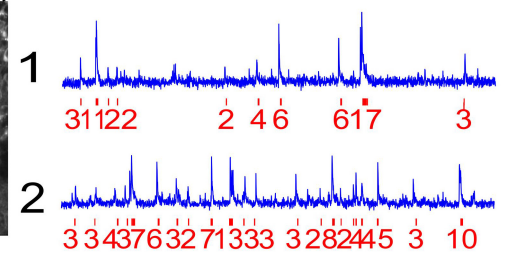

D

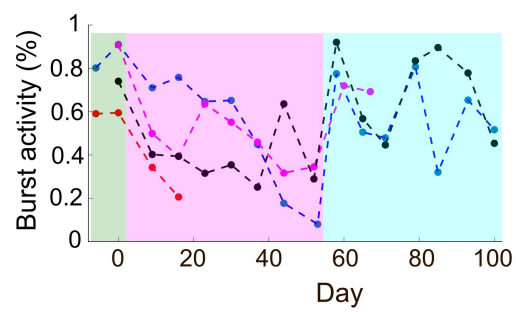

G

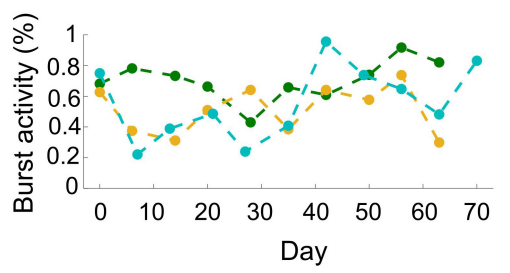

FIGURE 3 | Changes to CA1 activity patterns during demyelination and remyelination. (A) Time line of the demyelination/remyelination experiment for mice 1-4. Craniotomy surgery was performed between day -14 and -7 , and baseline activity recording from CA1 and DG neurons started between day -6 and day 0 [indicated by green background in panels (B-D)]. On day 0, after an activity recording session, we switched the diet to include 0.3\% cuprizone. This diet was administered until day 53 [demyelination period, indicated by magenta background in panels (B-D)], while we maintained weekly activity recordings. On day 53 , after an activity recording was completed, we switched the mice back to normal diet and monitored activity until day 100 [remyelination period, indicated by cyan background in panels (B-D)]. Bottom, two example fields of view acquired from mouse 1 (cuprizone group) showing the CA1 layer and activity of two CA1 example cells on days 53 and 58. The estimated timing and number of APs is shown (red) beneath each raw data trace (blue). (B) Summary of the average firing rates from all recorded CA1 neurons from mouse 1 during 107 days of recording. Firing rates decreased significantly from 0.345 to $0.17 \mathrm{~Hz}$ between day 0 and $9\left(P=1.8 \times 10^{-37}\right.$, Wilcoxon Rank Sum Test), after cuprizone diet had been introduced. Activity levels continued to significantly decrease down to zero on day 53 ( $P=0.002$ Mann-Kendall Trend Test), and were significantly increased to $0.205 \mathrm{~Hz}$ on day 58,5 days after normal diet was resumed $\left(P=4 \times 10^{-133}\right.$, Wilcoxon Rank Sum Test). Activity levels on the following days continued to increase compared to the demyelination period ( $P=0.064$, Mann-Kendall Trend Test), but remained lower than the pre-cuprizone period. Magenta and cyan backgrounds highlight the demyelination and remyelination periods, respectively; red lines correspond to medians, blue boxes show the 25th-75th percentile range, whisker length is the shorter of 1.5 times the 25th-75th range or the extreme data point. Outliers are not shown. ${ }^{* * *} P<0.001$. (C) Summary of the average firing rates from all recorded CA1 neurons from all cuprizone-treated mice. Solid lines connect the distribution medians, and the error bars indicate the 25th-75th percentile range for each recording session. For all recorded mice, there was a significant decrease in the average firing rate upon the start of cuprizone diet, between day 0 and 9 ( $n=4$ mice, $P<10^{-13}$, Wilcoxon Rank Sum Test), and a significant increase upon the termination of cuprizone diet, between day 53 and $58\left(n=3\right.$ mice, $P<10^{-32}$, Wilcoxon Rank Sum Test). Note that the rapid increase in firing rate on day 58 was followed by a second decrease in activity rate. ${ }^{* *} P<0.001$. (D) Summary of the fraction of cells that fired bursts of APs from all recorded cells for all cuprizone-treated mice. This fraction decreased for all mice during the demyelination period and rapidly recovered upon the start of the remyelination period. Colors are the same as in panel (C). $P$-values for the decrease were $0.035,0.108$, and 0.064 for mice 1, 2, 3, respectively (Mann-Kendall Trend Test). (E) Summary of the average firing rates from all recorded CA1 neurons from control mouse 5 , which received normal diet throughout the recording period, showing no similar decreases or increases in firing rate as those recorded from cuprizone-treated mice. (F) Summary of the average firing rates from all recorded CA1 neurons from all control mice. Solid lines connect the distribution medians, and the error bars indicate the 25th-75th percentile range for each recording session. None of the traces showed a significant monotonous trend ( $P=0.99,0.86,0.14$, for mice 5, 6, 7, respectively, Mann-Kendall Trend Test). (G) Summary of the fraction of cells that fired bursts of APs from all CA1-recorded neurons from all control mice. Colors are the same as in panel $\mathbf{( F )}$ 
5-7 between days 0 and 7, and days 0 and 49, respectively; see examples in Supplementary Figure 4). A repeated ANOVA test between the cuprizone and control groups yielded no statistically significant differences in the group and the interaction between the time and group, respectively $(P=0.03$ for the time effect, $P=0.77$ for the group effect, and $P=0.18$ for the grouptime interaction).

\section{Spontaneous Remyelination Restored the Activity of CA1 Excitatory Neurons}

It has been shown multiple times that once cuprizone diet is stopped and mice are returned to their normal diet, spontaneous remyelination begins (Skripuletz et al., 2008; Kipp et al., 2009; Dutta et al., 2013; Sachs et al., 2014). Therefore, once we stopped the cuprizone diet and returned the mice to normal diet, we kept monitoring the same mice during the remyelination period. We identified a substantial and significant increase in the average firing rate of individual mice within 5 days after the end of the cuprizone diet (1,356 and 1,207 neurons from $n=3$ mice recorded on days 53 and 58, respectively. $P<10^{-32}$ for the change in individual mice firing rate, Wilcoxon Rank Sum Test), but the group effect was not significant $(P=0.08$, paired $t$-test for change in median firing rates; Supplementary Movies 3, 6, see examples in Supplementary Figure 3). The amount of recovery was different across mice, but returned overall activity back to similar levels as the pre-cuprizone period $(-40.5 \%,+4.8 \%$, and $+104 \%$ of the pre-cuprizone median firing rate, $n=3$ mice, 1,135 and 1,207 neurons from days 0 to 58, respectively). Interestingly, this recovery was not monotonous (non-significant results in Mann-Kendall Trend Test for days 53-100), and included a second cycle of decreasing and increasing of CA1 cell activity (Figures 3B,C, days 65-70). We monitored cellular activity for 47 additional days following the termination of cuprizone diet, and identified partial recovery of cellular firing rates, to lower levels than those before the start of cuprizone diet (35 and 52\% of the activity level on day 0 , data from 807 and 1,201 neurons from $n=2$ mice, measured on day 0 and 100, respectively; data shown in Figures 3C,D, Supplementary Movie 7, see examples in Supplementary Figure 3).

\section{Cuprizone Diet Reduced Activity Levels of DG Excitatory Neurons}

For all tested mice, DG neuronal activity levels were lower than those of CA1 cells, and in the majority of DG neurons, firing of APs was not detected under our experimental conditions, similar to previously reported data (Pilz et al., 2016). In neurons that AP firing was detected, most fired a low number of APs. Therefore, we also quantified DG activity levels by assessing the fraction of cells that fired any number of APs, and the fraction of cells that fired at least one burst of more than five APs within a 660-ms time bin. Cuprizone diet caused a rapid decrease in the fraction of active cells by $49 \%$, and an $89 \%$ decrease in bursting activity, but insufficient group size resulted in non-significant $P$-values $(1,977$ and 2,326 neurons recorded on days 0 and 9 , respectively, $n=3$ mice; $P=0.19$ and $0.26, t$-test for change in fraction of active and bursting cells, respectively). Median firing rate was significantly decreased for two out of three mice $\left(P<10^{-15}\right.$ for mice 1 and 2, $P=0.338$ for mouse 3, Wilcoxon Rank Sum Test). This decrease in activity levels continued during the cuprizone diet period, similar to the trend detected in CA1 cells (Figures 4A,B, see examples in Supplementary Figure 5). On the last day of the cuprizone diet, the fraction of active cells was decreased to $15 \%$ of its value on day 0 (1,419 and 1,334 neurons from $n=2$ mice, recorded on day 0 and 53, respectively, $P<10^{-10}$ for change in median firing rate, Wilcoxon Rank Sum Test, Supplementary Movies 2, 8). Activity recording in control mice did not show such a decrease $(P=0.59$ and 0.30 , paired $t$-test for change in fraction of active and bursting cells between first and second recording dates, respectively; Figures 4C,D and Supplementary Movies 9, 10, see examples in Supplementary Figure 6).

\section{Spontaneous Remyelination Restored the Activity of DG Projection Neurons}

Dentate gyrus neurons presented a similar recovery pattern as CA1 neurons. DG cell activity increased rapidly upon termination of cuprizone diet, from 15 to $59 \%$ of the fraction of active cells on day $0(1,419,1,334$, and 1,059 cells from $n=2$ mice on days 0, 53, and 58, respectively, Supplementary Movie 11, see examples in Supplementary Figure 5). This was followed by a second decrease and a second increase in activity levels, to reach comparable levels to day 0 (78.3 and 220.2\% of fraction of active cells on day 0 for mice 1 and 3, respectively, data from 1,419 and 1,657 cells on day 0 and on the last day of recording, respectively; Figures 4A,B, Supplementary Movie 12, see examples in Supplementary Figure 6).

\section{DISCUSSION}

Repeating cycles of demyelination and remyelination are associated with the progression of MS in the majority of patients, who first experience the relapsing-remitting form of the disease (Chang et al., 2012). This highlights the necessity to understand the impact of these cycles on brain activity. In this study, we measured the effects of cuprizone diet, such as demyelination and remyelination, on hippocampal activity, which recapitulates the loss and partial recovery of myelin, consistently in the same brain regions and with a reliable and reproducible time course (Dutta et al., 2013; Sachs et al., 2014; Bai et al., 2016). We combined electrophysiology and functional microscopy for acute and long-term monitoring of the activity of projection neurons in CA1 and DG regions with cellular resolution over more than 100 days, during both the demyelination and remyelination phases. We identified cuprizone-induced deterioration of synaptic transmission of CA1 cells, and quantified the longitudinal effect of this deterioration on hippocampal activity in vivo. We report large changes in neuronal firing rates, with both fast and long-term components that followed the initiation and cessation of cuprizone diet. These changes were associated with the demyelination and remyelination processes, as were previously reported, and are in agreement with behavioral deficits and recovery that were also reported in cuprizone mice (Sachs et al., 2014; Bai et al., 2016), 

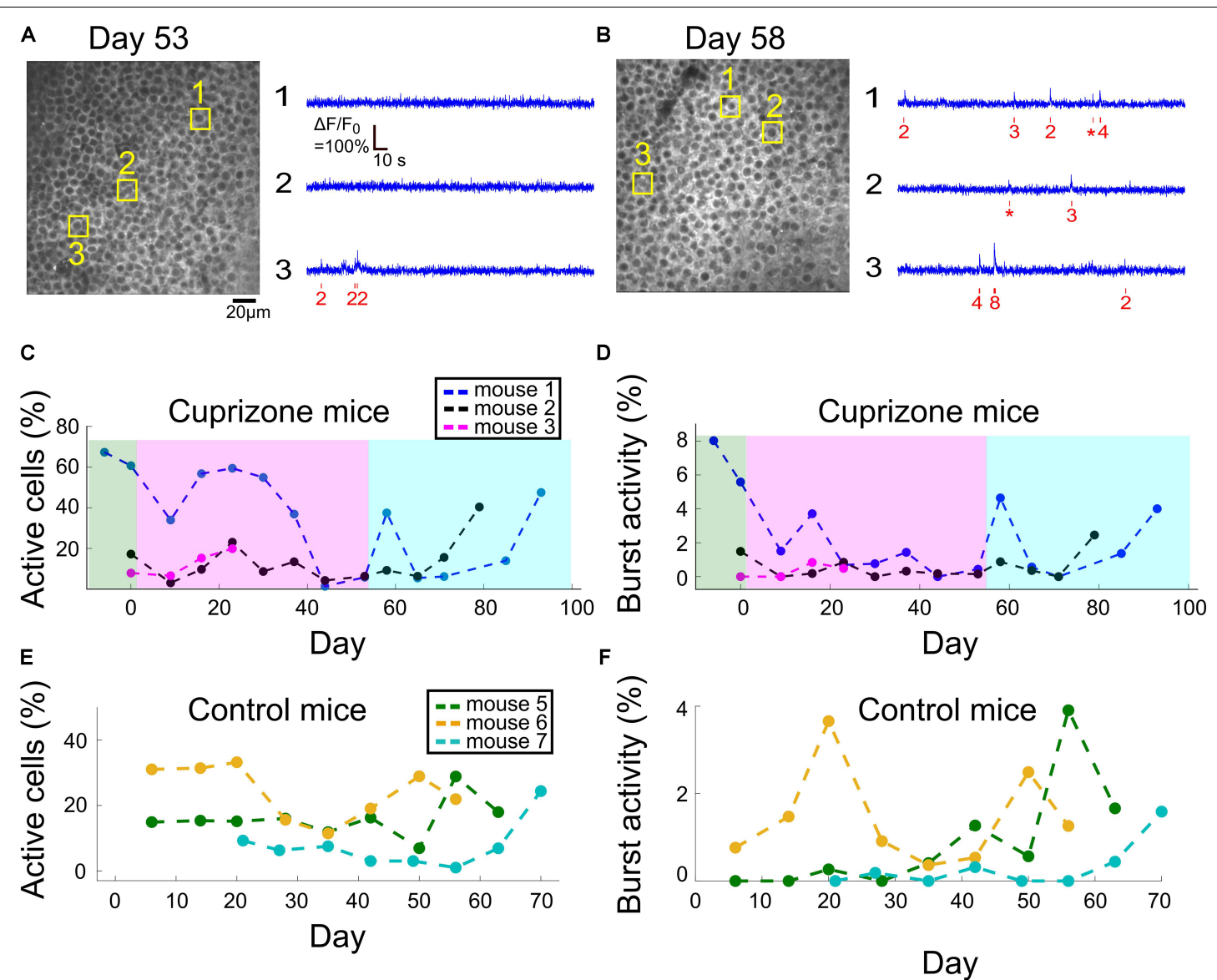

FIGURE 4 | Changes to DG activity patterns during demyelination and remyelination. (A) An example field of view of DG neurons from mouse 1, after 53 days of cuprizone diet, with activity traces of three example cells. The estimated timing and number of APs is shown (red) beneath each raw data trace (blue). (B) An example field of view of DG neurons from mouse 1, 5 days after the end of the cuprizone diet, with activity traces of three example cells. The estimated timing and number of APs is shown (red) beneath each raw data trace (blue). (C) Summary of the fraction of active DG cells from all recorded DG cells for all cuprizone-treated mice. The low-level recorded activity of DG cells is in line with previous findings (Pilz et al., 2016). However, the cuprizone diet reduced the activity even further, and the termination of cuprizone diet was followed by a noticeable recovery. DG cells of mouse 3 could be recorded only during the first four recording sessions. (D) Summary of the fraction of DG cells that fired bursts of APs from all recorded DG cells in cuprizone-treated mice shows a similar trend to the change in CA1 activity patterns. Colors are the same as in panel (C). (E) Summary of the fraction of active DG cells from all recorded DG cells for all control mice. (F) Summary of the fraction of DG cells that fired bursts of APs from all recorded DG cells in control mice. Colors are the same as in panel (E).

as well as changes to the excitability levels of single neurons (Praet et al., 2014; Hamada et al., 2017) and disrupted large-scale activity patterns (Hoffmann et al., 2008).

The time-scales of cuprizone-mediated brain activity degeneration, and recovery once cuprizone diet was stopped, are in agreement with previous studies (Sachs et al., 2014; Bai et al., 2016). However, our recordings have identified rapid changes and non-monotonous trends for both conditions that have not yet been reported. A large fraction of the activity change occurred within 5-9 days (one recording session) from the initiation and cessation of cuprizone diet, faster than the reported time scale of 3-6 weeks for demyelination and remyelination (Sachs et al., 2014; Bai et al., 2016). Interestingly, this relatively rapid change was followed by a "rebound" change in activity levels, i.e., the activity increased during cuprizone diet, and decreased during remyelination. Increased activity during demyelination, approximately 3-6 weeks after the start of cuprizone diet, may be explained by partial remyelination due to maturation of the oligodendrocyte progenitor cells into mature myelinating oligodendrocytes (Sachs et al., 2014), but other characteristics of the effects of cuprizone on neuronal and non-neuronal cells, and how it translates to the reported rapid changes in activity, will require additional studies. It is possible that cuprizone has, in addition to the reported effect on oligodendrocytes, a direct effect on neuronal health, or alternatively an indirect effect mediated by glial cells, that resulted in the rapid changes we identified. In addition, this study included both male and female mice, and the reduction in activity levels seemed to be stronger in males (mice 1 and 4). Though the number of mice used in this study does not allow for a definitive assessment of sex-specific differences, such differences may be expected based upon the existing findings (Kipp et al., 2009; Ohgomori and Jinno, 2019). 
In agreement with fluorescent imaging results, electrophysiological recording of CA1 synaptic transmission showed significant depression starting at 3 weeks of demyelination, which is earlier than previously reported (Sachs et al., 2014). Depression of EPSPs progressed on a weekly basis, reaching a nadir level by 6 weeks of demyelination. Interestingly, AVs were well preserved, even in 6-week demyelinated slices, suggesting that the primary site of failure for synaptic transmission is post-synaptic. Indeed, cuprizone-induce demyelination reduces AMPA receptors in the CA1 region of hippocampus (Dutta et al., 2013). Additionally, deficits in excitatory synaptic transmission during experimental autoimmune encephalomyelitis (EAE) correlated with disruption of PSD-95 integrity involving both AMPA and NMDA receptormediated currents (Ziehn et al., 2012a,b) while preserving presynaptic function in CA1 neurons. In contrast, recordings in layer $\mathrm{V}$ pyramidal neurons in somatosensory cortex of mice that were kept on cuprizone diet for 5 weeks revealed that AP propagation switched from rapid saltatory conduction toward a slow continuous wave broadening the presynaptic AP, with reduced velocity presumably due to redistribution of fast-acting K channels (Hamada et al., 2017). Furthermore, AP changes were accompanied by demyelination of internodes, variable reorganization of nodal domains, and frequent sprouting of axons. Characterizing the structural changes in our animal model will require additional work. However, taken together, it seems that loss of myelin in gray matter causes a wide range of site-specific structural and functional changes.

Optical activity monitoring allows probing how connected brain regions are affected by external input, and how they affect each other. We recorded activity both from CA1 and DG neurons in the same mice, and identified substantial differences in their activity patterns (Figures 2-4), in agreement with previous studies (Ziv et al., 2013; Pilz et al., 2016). The deep-tissue location and low level of activity of DG neurons under anesthesia make them more challenging to monitor compared to CA1 neurons (Supplementary Figure 2). Therefore, more studies are required to obtain a better understanding of cuprizoneinduced changes in DG cells, preferably in awake mice, where activity levels are expected to be higher (Pilz et al., 2016). Nevertheless, longitudinal DG recording is feasible using laser power that is not expected to produce substantial heating or thermal damage to the tissue (Podgorski and Ranganathan, 2016). Our electrophysiological studies have highlighted that cellular excitability of CA1 neurons was interrupted during cuprizone-induced demyelination, suggesting impaired synaptic transmission, which was restored during remyelination. Future studies may characterize how DG pre- and post-synaptic activity is modulated, and how it affects the CA1 pre-synaptic signal via feed-forward hippocampal circuitry. Such studies may reveal whether cuprizone-mediated diet attenuates activity in other brain regions, and hence also the input to the hippocampus, reduces the excitability of DG neurons via changes in excitationinhibition balance, or similarly affects cellular excitability in both CA1 and DG neurons.

A limitation of the in vivo approach we used in this study is the relatively low number of mice in each group, which is related to the amount of time required to record and analyze the activity data from each mouse. The small group size limited the significance of the statistical analyses that we performed. We received significant $P$-values for changes in activity characteristics from the same animal, which relied on the large number of cells that we sampled. However, our statistical analyses were less conclusive for $t$-tests across median values from mice in the same group, and were non-significant for a repeated ANOVA across the cuprizone and control groups. A preferred approach for overcoming this limitation is to increase the group sizes, which might require adopting an automatic analysis pipeline for improving the throughput of such experiments (Pachitariu et al., 2017).

Finally, applying optical methods for monitoring the brain offers an attractive approach for acquiring essential in vivo information to link structure, function, and behavior. We demonstrated that TPLSM recording of GECI signal enables direct evaluation of functional properties of neurons in a mouse model of MS-like symptoms. Moreover, we show that deterioration of synaptic transmission in CA1 cells is reflected in the activity levels of these neurons, and can be measured in vivo in a longitudinal manner. Future studies may combine this approach with emerging methods for in vivo measurements of the myelin sheath using label-free third harmonic generation (Farrar et al., 2011; Redlich and Lim, 2019) to allow for all-optical detection of demyelination and activity levels in the same cells. Complementary behavioral data can be acquired with standard behavioral and cognitive tests for assessing the mouse condition, such as the Morris water maze (Dutta et al., 2013). Such experimental platforms will provide a more holistic assessment of the mouse condition. Such an experimental platform may also be used as an evaluation testbed for comparing the efficacy of new pharmaceutical intervention treatments for protecting synaptic transmission and neuronal activity patterns, reducing demyelination levels, and/or relieving behavioral deficits.

\section{DATA AVAILABILITY STATEMENT}

The datasets generated for this study are available on request to the corresponding author.

\section{ETHICS STATEMENT}

The animal study was reviewed and approved by the Lerner Research Institute, Institutional Animal Care and Use Committee and Institutional Biosafety Committee.

\section{AUTHOR CONTRIBUTIONS}

$\mathrm{HD}, \mathrm{BT}$, and $\mathrm{SB}$ initiated the project. $\mathrm{SB}, \mathrm{CB}$, and LT conducted the electrophysiological recording experiments and their analysis. $\mathrm{AD}$ conducted the optical recording experiments. AD, JS, TD, and HD performed the optical data analysis. HD and SB wrote the manuscript with comments from the other authors. 


\section{FUNDING}

This research was partially funded by an MS Society Pilot Grant (PP-1901-33093) to HD and NIH grants (R35NS09730) to BT and (AG033720) to SB.

\section{ACKNOWLEDGMENTS}

We thank the HHMI Janelia GENIE project for developing and sharing the Thyl-jRGECOla transgenic mice. We thank Dr. Vanzetta and his colleagues for developing and sharing the AP extraction model used in this study. We would like to thank Drs. Albert Lee and Jae Sung Lee for sharing their hippocampal

\section{REFERENCES}

Bai, C. B., Sun, S., Roholt, A., Benson, E., Edberg, D., and Medicetty, S. (2016). A mouse model for testing remyelinating therapies. Exp. Neurol. 283, 330-340. doi: 10.1016/j.expneurol.2016.06.033

Chang, A., Staugaitis, S. M., Dutta, R., Batt, C. E., Easley, K. E., and Chomyk, A. M. (2012). Cortical remyelination: a new target for repair therapies in multiple sclerosis. Ann. Neurol. 72, 918-926. doi: 10.1002/ana.23693

Chari, D. M. (2007). Remyelination in multiple sclerosis. Int. Rev. Neurobiol. 79, 589-620. doi: 10.1016/s0074-7742(07)79026-8

Chen, T. W., Wardill, T. J., Sun, Y., Pulver, S. R., Renninger, S. L., and Baohan, A. (2013). Ultrasensitive fluorescent proteins for imaging neuronal activity. Nature 499, 295-300. doi: 10.1038/nature 12354

Chiaravalloti, N. D., and DeLuca, J. (2008). Cognitive impairment in multiple sclerosis. Lancet Neurol. 7, 1139-1151.

Dana, H., Chen, T.-W., Hu, A., Shields, B. C., Guo, C., and Looger, L. (2014). Thy1GCaMP6 transgenic mice for neuronal population imaging in vivo. PLoS One 9:e108697. doi: 10.1371/journal.pone.0108697

Dana, H., Mohar, B., Sun, Y., Narayan, S., Gordus, A., and Hasseman, J. P. (2016). Sensitive red protein calcium indicators for imaging neural activity. eLife 5:e12727. doi: 10.7554/eLife.12727

Dana, H., Novak, O., Guardado-Montesino, M., Fransen, J. W., Hu, A., and Borghuis, B. G. (2018). Thyl transgenic mice expressing the red fluorescent calcium indicator jRGECO1a for neuronal population imaging in vivo. PLoS One 13:e0205444. doi: 10.1371/journal.pone.0205444

Dana, H., Sun, Y., Mohar, B., Hulse, B. K., Kerlin, A. M., and Hasseman, J. P. (2019). High-performance calcium sensors for imaging activity in neuronal populations and microcompartments. Nat. Methods 16, 649-657. doi: 10.1038/ s41592-019-0435-6

Danielson, N. B., Kaifosh, P., Zaremba, J. D., Lovett-Barron, M., Tsai, J., and Denny, C. A. (2016). Distinct contribution of adult-born hippocampal granule cells to context encoding. Neuron 90, 101-112. doi: 10.1016/j.neuron.2016.02.019

Danielson, N. B., Turi, G. F., Ladow, M., Chavlis, S., Petrantonakis, P. C., Poirazi, P., et al. (2017). In vivo imaging of dentate gyrus mossy cells in behaving mice. Neuron 93, 552.e4-559.e4. doi: 10.1016/j.neuron.2016.12.019

Deneux, T., Kaszas, A., Szalay, G., Katona, G., Lakner, T., and Grinvald, A. (2016). Accurate spike estimation from noisy calcium signals for ultrafast threedimensional imaging of large neuronal populations in vivo. Nat. Commun 7:12190. doi: 10.1038/ncomms 12190

Dombeck, D. A., Harvey, C. D., Tian, L., Looger, L. L., and Tank, D. W. (2010). Functional imaging of hippocampal place cells at cellular resolution during virtual navigation. Nat. Neurosci. 13, 1433-1440. doi: 10.1038/nn.2648

Dong, H. W. (2008). The Allen Reference Atlas: A Digital Color Brain Atlas of the C57Bl/6J Male Mouse. Hoboken, NJ: John Wiley \& Sons Inc.

Dutta, R., Chomyk, A. M., Chang, A., Ribaudo, M. V., Deckard, S. A., and Doud, M. K. (2013). Hippocampal demyelination and memory dysfunction are associated with increased levels of the neuronal microRNA miR-124 and reduced AMPA receptors. Ann. Neurol. 73, 637-645. doi: 10.1002/ana.23860 window implantation technique, Drs. Ranjan Dutta, Chris Nelson, and Dimitrios Davalos for reviewing the manuscript and for their insightful comments, and Sarah Stanko and Anthony Chomyk for assisting with mouse husbandry. Materials and data were provided by the Cleveland Clinic Foundation (CCF). All rights, title, and interest in the materials and data are owned by CCF.

\section{SUPPLEMENTARY MATERIAL}

The Supplementary Material for this article can be found online at: https://www.frontiersin.org/articles/10.3389/fncel. 2019.00588/full\#supplementary-material

Farrar, M. J., Wise, F. W., Fetcho, J. R., and Schaffer, C. B. (2011). In vivo imaging of myelin in the vertebrate central nervous system using third harmonic generation microscopy. Biophys. J. 100, 1362-1371. doi: 10.1016/j.bpj.2011. 01.031

Geurts, J. J., and Barkhof, F. (2008). Grey matter pathology in multiple sclerosis. Lancet Neurol. 7, 841-851.

Goldenberg, M. M. (2012). Multiple sclerosis review. Pharm. Therap. 37, 175-184.

Hamada, M. S., Popovic, M. A., and Kole, M. H. P. (2017). Loss of saltation and presynaptic action potential failure in demyelinated axons. Front. Cell. Neurosci. 11:45. doi: 10.3389/fncel.2017.00045

Hoffmann, K., Lindner, M., Gröticke, I., Stangel, M., and Löscher, W. (2008). Epileptic seizures and hippocampal damage after cuprizone-induced demyelination in C57BL/6 mice. Exp. Neurol. 210, 308-321. doi: 10.1016/j. expneurol.2007.11.005

Huebner, N. S., Mechling, A. E., Lee, H.-L., Reisert, M., Bienert, T., and Hennig, J. (2017). The connectomics of brain demyelination: functional and structural patterns in the cuprizone mouse model. Neuroimage 146, 1-18. doi: 10.1016/j. neuroimage.2016.11.008

Kipp, M., Clarner, T., Dang, J., Copray, S., and Beyer, C. (2009). The cuprizone animal model: new insights into an old story. Acta Neuropathol. 118, 723-736. doi: 10.1007/s00401-009-0591-3

Koutsoudaki, P. N., Skripuletz, T., Gudi, V., Moharregh-Khiabani, D., Hildebrandt, H., Trebst, C., et al. (2009). Demyelination of the hippocampus is prominent in the cuprizone model. Neurosci. Lett. 451, 83-88. doi: 10.1016/j.neulet.2008. 11.058

Lee, J. S., Briguglio, J., Romani, S., and Lee, A. K. (2019). The statistical structure of the hippocampal code for space as a function of time, context, and value. bioRxiv[Preprint]

Madisen, L., Garner, A. R., Shimaoka, D., Chuong, A. S., Klapoetke, N. C., and Li, L. (2015). Transgenic mice for intersectional targeting of neural sensors and effectors with high specificity and performance. Neuron $85,942-958$. doi: 10.1016/j.neuron.2015.02.022

Mahad, D. H., Trapp, B. D., and Lassmann, H. (2015). Pathological mechanisms in progressive multiple sclerosis. Lancet Neurol. 14, 183-193. doi: 10.1016/s14744422(14)70256-x

Ohgomori, T., and Jinno, S. (2019). Cuprizone-induced demyelination in the mouse hippocampus is alleviated by phytoestrogen genistein. Toxicol. Appl. Pharmacol. 363, 98-110. doi: 10.1016/j.taap.2018.11.009

Ouzounov, D. G., Wang, T., Wang, M., Feng, D. D., Horton, N. G., and CruzHernández, J. C. (2017). In vivo three-photon imaging of activity of GCaMP6labeled neurons deep in intact mouse brain. Nat. Methods 14, 388-390. doi: $10.1038 /$ nmeth.4183

Pachitariu, M., Stringer, C., Dipoppa, M., Schröder, S., Rossi, L. F., and Dalgleish, H. (2017). Suite2p: beyond 10,000 neurons with standard two-photon microscopy. bioRxiv[Preprints]

Pilz, G.-A., Carta, S., Stäuble, A., Ayaz, A., Jessberger, S., and Helmchen, F. (2016). Functional imaging of dentate granule cells in the adult mouse hippocampus. J. Neurosci. 36, 7407-7414. doi: 10.1523/JNEUROSCI.3065-15.2016 
Podgorski, K., and Ranganathan, G. (2016). Brain heating induced by near-infrared lasers during multiphoton microscopy. J. Neurophysiol. 116, 1012-1023. doi: 10.1152/jn.00275.2016

Praet, J., Guglielmetti, C., Berneman, Z., Van der Linden, A., and Ponsaerts, P. (2014). Cellular and molecular neuropathology of the cuprizone mouse model: clinical relevance for multiple sclerosis. Neurosci. Biobehav. Rev. 47, 485-505. doi: 10.1016/j.neubiorev.2014.10.004

Provencio, J. J., Swank, V., Lu, H., Brunet, S., Baltan, S., and Khapre, R. V. (2016). Neutrophil depletion after subarachnoid hemorrhage improves memory via NMDA receptors. Brain Behav. Immun. 54, 233-242. doi: 10.1016/j.bbi.2016. 02.007

Redlich, M. J., and Lim, H. (2019). A method to measure myeloarchitecture of the murine cerebral cortex in vivo and ex vivo by intrinsic third-harmonic generation. Front. Neuroanat. 13:65. doi: 10.3389/fnana.2019.00065

Sachs, H. H., Bercury, K. K., Popescu, D. C., Narayanan, S. P., and Macklin, W. B. (2014). A new model of cuprizone-mediated demyelination/remyelination. ASN Neuro 6:1759091414551955. doi: 10.1177/1759091414551955

Skripuletz, T., Lindner, M., Kotsiari, A., Garde, N., Fokuhl, J., and Linsmeier, F. (2008). Cortical demyelination is prominent in the murine cuprizone model and is strain-dependent. Am. J. Pathol. 172, 1053-1061. doi: 10.2353/ajpath. 2008.070850

Staffen, W., Mair, A., Zauner, H., Unterrainer, J., Niederhofer, H., and Kutzelnigg, A. (2002). Cognitive function and fMRI in patients with multiple sclerosis: evidence for compensatory cortical activation during an attention task. Brain 125, 1275-1282. doi: 10.1093/brain/awf125

Svoboda, K., and Yasuda, R. (2006). Principles of two-photon excitation microscopy and its applications to neuroscience. Neuron 50, 823-839. doi: 10.1016/j.neuron.2006.05.019

Tekkok, S., and Krnjevic, K. (1995). Long-term potentiation in hippocampal slices induced by temporary suppression of glycolysis. J. Neurophysiol. 74, 2763-2766. doi: 10.1152/jn.1995.74.6.2763

Thevenaz, P., Ruttimann, U. E., and Unser, M. (1998). A pyramid approach to subpixel registration based on intensity. IEEE Trans Image Process 7, 27-41. doi: $10.1109 / 83.650848$

Vega-Riquer, J. M., Mendez-Victoriano, G., Morales-Luckie, R. A., and GonzalezPerez, O. (2019). Five decades of cuprizone, an updated model to replicate demyelinating diseases. Curr. Neuropharmacol. 17, 129-141. doi: 10.2174/ 1570159X15666170717120343

Wallin, M. T., Culpepper, W. J., Campbell, J. D., Nelson, L. M., Langer-Gould, A., and Marrie, R. A. (2019). The prevalence of MS in the United States. A population-based estimate using health claims data. Neurology 92, e1029e1040. doi: 10.1212/wnl.0000000000007035

Wekselblatt, J. B., Flister, E. D., Piscopo, D. M., and Niell, C. M. (2016). Largescale imaging of cortical dynamics during sensory perception and behavior. J. Neurophysiol. 115, 2852-2866. doi: 10.1152/jn.01056.2015

Xu, H., Yang, H.-J., Zhang, Y., Clough, R., Browning, R., and Li, X.-M. (2009). Behavioral and neurobiological changes in C57BL/6 mice exposed to cuprizone. Behav. Neurosci. 123, 418-429. doi: 10.1037/a0014477

Ziehn, M. O., Avedisian, A. A., Dervin, S. M., O'dell, T. J., and Voskuhl, R. R. (2012a). Estriol preserves synaptic transmission in the hippocampus during autoimmune demyelinating disease. Lab. Invest. 92, 1234-1245. doi: 10.1038/ labinvest.2012.76

Ziehn, M. O., Avedisian, A. A., Dervin, S. M., Umeda, E. A., O’Dell, T. J., and Voskuhl, R. R. (2012b). Therapeutic testosterone administration preserves excitatory synaptic transmission in the hippocampus during autoimmune demyelinating disease. J. Neurosci. 32, 12312-12324. doi: 10.1523/JNEUROSCI. 2796-12.2012

Ziv, Y., Burns, L. D., Cocker, E. D., Hamel, E. O., Ghosh, K. K., and Kitch, L. J. (2013). Long-term dynamics of CA1 hippocampal place codes. Nat. Neurosci. 16, 264-266. doi: 10.1038/nn.3329

Conflict of Interest: The authors declare that the research was conducted in the absence of any commercial or financial relationships that could be construed as a potential conflict of interest.

Copyright (c) 2020 Das, Bastian, Trestan, Suh, Dey, Trapp, Baltan and Dana. This is an open-access article distributed under the terms of the Creative Commons Attribution License (CC BY). The use, distribution or reproduction in other forums is permitted, provided the original author(s) and the copyright owner(s) are credited and that the original publication in this journal is cited, in accordance with accepted academic practice. No use, distribution or reproduction is permitted which does not comply with these terms. 